\title{
The interplay between spatial and heliconical orientational order in twist-bend nematic materials
}

\author{
R. Saha ${ }^{3}$, C. Feng ${ }^{1,2}$, C. Welch ${ }^{4}$, G. H. Mehl ${ }^{4}$, J. Feng ${ }^{2}$, C. Zhu ${ }^{2}$, J. Gleeson ${ }^{3}$, S. Sprunt ${ }^{1,3}$ and \\ A. Jákli ${ }^{1,3^{*}}$ \\ ${ }^{1}$ Chemical Physics Interdisciplinary Program, Advanced Materials and Liquid Crystal Institute, \\ Kent State University, Kent, $\mathrm{OH} 44242$, USA \\ ${ }^{2}$ Advanced Light Source, Lawrence Berkeley National Lab, Berkeley, CA 94720, USA \\ ${ }^{3}$ Department of Physics, Kent State University, Kent, OH 44242, USA \\ ${ }^{4}$ Department of Chemistry, University of Hull, Hull, UK
}

\begin{abstract}
:
The helical pitch formed by organic molecules, such as the $\alpha$-helix of proteins, usually requires hydrogen bonding between chiral units and long-range positional order. It was recently found that certain liquid crystal oligomers can have a twist-bend nematic $\left(N_{T B}\right)$ phase with nanoscale heliconical structure without hydrogen bonding, molecular chirality or positional order.

To understand the nature of this unique structure, here we present hard and resonant tender $X$-ray scattering studies of two novel sulfur containing dimer materials. We simultaneously measure the temperature dependences of the helical pitch and the correlation length of both the helical and positional order. In addition to an unexpected strong variation of the pitch with the length of the spacer connecting the monomer units, we find that at the transition to the $N_{T B}$ phase the positional correlation length drops. The helical structure was found not only in the $N_{T B}$ phase but observed even in the upper range of a smectic phase that forms just below the $N_{T B}$ state. The coexistence of smectic layering and the heliconical order indicates a layered (SmATB) phase wherein the rigid units of the dimers are tilted with respect to the smectic layer normal in order to accommodate the bent conformation of the dimers and the tilt direction rotates along the heliconical axis.
\end{abstract}




\section{Introduction}

The formation of helical structure in molecular systems usually requires crystal or liquid crystal phases with chiral components. The smallest helical pitch formed by organic molecules is the $\alpha$-helix of proteins ${ }^{1}$ with $p=0.55 \mathrm{~nm}$, meaning 3.6 amino acids in L-configuration make one turn. ${ }^{1}$ Such a tight pitch requires internal hydrogen bonding between chiral amino acid residues that join together in peptide chains that crystallize into a structure with long-range positional order. The helical pitch of chiral nematic liquid crystals (3-D anisotropic fluids) of rod-shaped molecules ranges from a tenth of a micrometer to several hundred micrometers, i.e., hundreds to thousands of chiral molecules are needed to make one turn. ${ }^{2}$ Recently achiral liquid crystal oligomers (dimers ${ }^{3-19}$, trimers ${ }^{20,21}$ and tetramers ${ }^{12,21,22}$ ) with rigid arms connected by odd-numbered methylene or ether groups were found to exhibit a twist-bend nematic phase $\left(\mathrm{N}_{\mathrm{TB}}\right)^{23-25}$, which features a heliconical structure with nanoscale pitch, $\mathrm{p}=6-20 \mathrm{~nm}$ range ${ }^{6,26}$. Remarkably such a helical structure requires neither hydrogen bonding, nor molecular chirality or positional order. The application of electrical fields can lead to an unwinding of the helix and the formation of the socalled splay bend phase. ${ }^{27}$

Although a number of theoretical models have been developed to describe the heliconical phase structure, important features are still quite poorly understood. Indeed, the very nature of the phase is still controversial. ${ }^{28,29}$ Questions range from fundamental issues such as the thermodynamic order of the phase transition to the high temperature nematic phase, the unwinding of the helix with temperature, the nature of the very short pitch of the helix and, crucially, why the rigid extended linear aromatic cores do not nano-segregate from the flexible alkyl chains to form layers with positional ordering instead of building a highly correlated structure with orientational ordering. Models using continuum theory ${ }^{23,30}$ predict that the pitch diverges as $p \propto\left(T_{C}-T\right)^{-1 / 2}$, where the difference between the critical temperature $T_{C}$ and the $N_{T B}$ phase transition temperature $T_{T B}\left(T_{C}-T_{T B} \sim 1{ }^{\circ} \mathrm{C}\right)$ is related to the molecular bend and flexoelectric coupling. ${ }^{31}$ An alternative theoretical approach is based on microscopic molecular parameters, such as the bend and twist angles ( $\beta$ and $\alpha$ ) between the molecular arms, and on pair-pair correlations. ${ }^{32,33}$ Small differences in dipole moments and in the linearity of the mesogenic groups of the molecules impact massively the formation and the pitch of the helices, the tilt of the mesogens, and concomitantly elemental 
parameters of the helical structure such as the radius and contour lengths. For all these questions the correlation with molecular parameters, such as molecular interdigitation and bend angles, are crucial to arrive at a full understanding of the phase.

The first proof of the nanoscale pitch was provided by freeze fracture transmission electron microscopy (FF-TEM) measurements. ${ }^{6,26}$ As they require rapid quenching of samples from welldefined different temperatures, detailed temperature dependence measurements would require large number of samples. The temperature dependence of the orientation (bond) ordering using only one sample was first probed with resonant soft X-ray scattering at carbon K-edge (RSoXS) 34-37. Although RSoXS can be employed for all materials that contain carbon atoms, the $\lambda=4.4 \mathrm{~nm}$ wavelength of the soft x-ray limits the resolution to a few nanometers and its $l \sim 0.3 \mu \mathrm{m}$ penetration depth requires the preparation of submicron thick films. ${ }^{38}$ For dimer materials containing sulfur atom(s) Tender Resonant X-ray Scattering (TReXS) at the sulfur K-edges ( $\mathrm{E}=2.471 \mathrm{keV}, \lambda \sim 0.5 \mathrm{~nm}$, $l \sim 10 \mu \mathrm{m}$ ) offers a more attractive alternative for precise measurement of the temperature dependence of the helical pitch of the $\mathrm{N}_{\text {Тв }}$ phase, as shown by several recent studies. ${ }^{39-44}$

In spite of the fact that small angle hard X-ray scattering is not suitable for pitch measurements due to the lack of an electron density modulation coupled to the heliconical structure, recent studies showed that careful analysis of synchrotron SAXS results can provide important information about the molecular associations both in the $\mathrm{N}_{\text {TB }}$ phase and the $\mathrm{N}$ phase above it. ${ }^{22}$ Combining SAXS and TReXS measurements allows one to probe the relation between short-range positional and longer range heliconical orientational order in the $\mathrm{N}_{\text {Tв }}$ phase.

In this paper we combine SAXS and TReXS measurements on two novel sulfur-containing analogues of fluorinated dimers with $n$-pentyl $\left(\mathrm{C}_{5} \mathrm{H}_{11}\right)$ terminal chains. ${ }^{45,46}$ In addition to the temperature dependences of the periodicities of the molecular associations and of the heliconical pitch, we also measure correlation lengths of the positional order of the molecular associations and of the heliconical orientational order. We find that below the N-N $\mathrm{N}_{\mathrm{TB}}$ transition the positional order decreases to about $6 \mathrm{~nm}$, while the heliconical-order increases to about $60 \mathrm{~nm}$. These results lead us to propose a refined packing model of the heliconical structure that can explain both the reduction in positional correlations and the temperature dependence of the heliconical pitch. We also show that there approximately $4^{\circ} \mathrm{C}$ temperature range below the $\mathrm{N}_{\text {Tв }}$ phase where the smectic order and the heliconical order coexist, and we discuss the possibility of a nematic to smectic twistbend phase transition occurring in the system. 


\section{Results and discussion}

\section{A. $\quad$ Materials}

The synthesis towards the investigated materials A (Butyl(4"-(7-(2',3'-difluoro-4"-pentyl[1,1':4',1"-terphenyl]-4-yl)heptyl)-2',3'-difluoro-[1,1':4',1"-terphenyl]-4-yl)sulfane) and $\mathbf{B}$ (butyl(4"-(11-(2',3'-difluoro-4"-pentyl-[1,1':4',1"-terphenyl]-4-yl)undecyl)-2',3'-difluoro$\left[1,1^{\prime}: 4\right.$ ', 1"-terphenyl]-4-yl)sulfane) is shown in Figure 1. The molecules $\mathbf{A}$ and $\mathbf{B}$ are characterized by two aromatic difluoroterphenyl units separated either by a heptyl or an undecyl spacer. The aromatic units are terminated on one side either by a pentyl group linked directly to the aromatic core or a butylthioether function, introducing the functionality for TReXS measurements. ${ }^{31}$ The architectures are as close as possible to $\mathrm{DTCnCm}$ dimers reported earlier. ${ }^{45,46}$ Notable is that for those materials it was not possible to obtain RSoXS results, probably due to the large number of different carbon atoms in the flexible terminal chains. The synthesis of $\mathbf{A}$ and $\mathbf{B}$ follows routes described previously and recently reported in detail by Stevenson et al. ${ }^{47}$ New synthetic steps are associated with the formation of 4'-(butylthio)-2,3-difluoro-[1,1'-biphenyl]-4-yl)boronic acid (2), obtained from butyl(2',3'-difluoro-[1,1'-biphenyl]-4-yl)sulfane by a reaction with $n$-Butylithium at $-78^{\circ} \mathrm{C}$ under an $\mathrm{N}_{2}$ atmosphere followed by an addition of trimethyl borate and subsequent acidification at room temperature by addition of $10 \% \mathrm{HCl}$. 2 was reacted in transition metal catalyst $\left(\mathrm{Pd}\left(\mathrm{PPH}_{3}\right)_{4}\right)$ mediated coupling reactions with either $\mathbf{1 a}$ or $\mathbf{1} \mathbf{b}$ to obtain the target materials $\mathbf{A}$ and $\mathbf{B}$ in yields after purification of respectively $71 \%$ and $68 \%$.

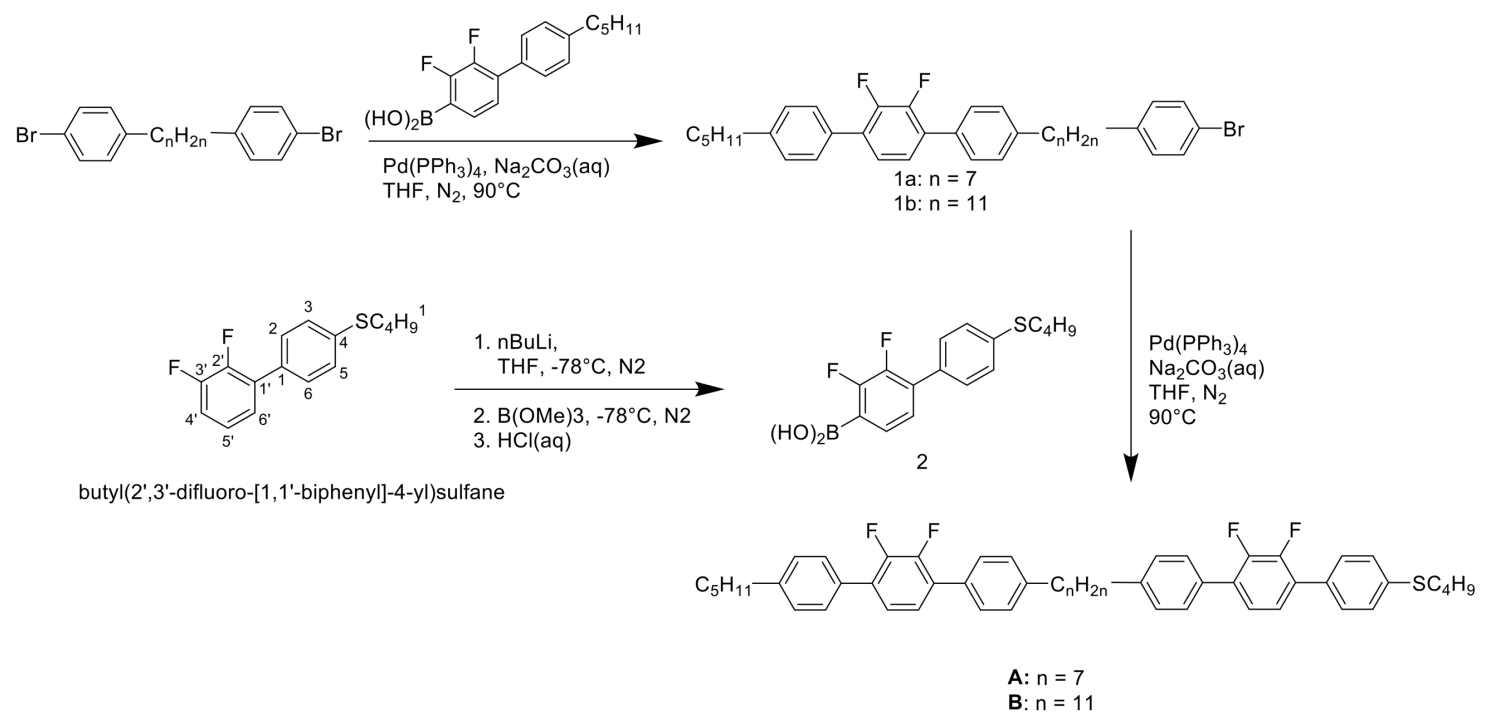

Figure 1: Scheme of the Synthesis of the investigated systems $\boldsymbol{A}$ and $\boldsymbol{B}$. 
Details of the synthesis are provided in the Electronic Supporting Information (ESI).

The phase transition temperatures in ${ }^{\circ} \mathrm{C}$ as determined by DSC measurements on heating and cooling with $10^{\circ} \mathrm{C} / \mathrm{deg}$ rate are as follows. A: $\mathrm{Cr} 130.5$ (SmA 109.3 N ${ }_{\mathrm{TB}}$ 122.5) N 144.8 Iso; B: Cr 90.5 SmA 107.5 $\mathrm{N}_{\mathrm{TB}} 125.0 \mathrm{~N} 161.7$ Iso. Phases in parenthesis are monotropic, i.e., appear only on cooling from the isotropic phase. These transition temperatures are different from that of analogues where the thiol group is substituted for a methylene group as follows. Compared to DTC5C7, which is analogue to $\mathbf{A}$ the melting point is higher by about $30{ }^{\circ} \mathrm{C}$ and the clearing temperature is lower by $12^{\circ} \mathrm{C}$, the $N-N_{t b}$ transition by $5^{\circ} \mathrm{C}$. In DTC5C7 the sample needs to be supercooled to 76 ${ }^{\circ} \mathrm{C}$ to detect a smectic phase. DTC5C11, which is analogue to $\mathrm{B}$, has no smectic phase, but melts at $97.8^{\circ} \mathrm{C}$ to the $\mathrm{N}_{\text {tb }}$ phase, transits to the $\mathrm{N}$ phase at $127.9^{\circ} \mathrm{C}$ and turns isotropic at $165.8^{\circ} \mathrm{C} . .^{46,47}$

\section{B. $\quad$ Polarized Optical Microscopy (POM)}

Figure 2 shows the polarized optical microscopy (POM) textures of $5 \mu \mathrm{m}$ cells with planar alignment after cooling from the isotropic phase with $1{ }^{\circ} \mathrm{C} / \mathrm{min}$ rate. Figure 2 (a-d) and (e-h) show the textures of $\mathbf{A}$ and $\mathbf{B}$, respectively. The uniform textures ( $a$ and e) represent aligned uniaxial nematic phase at $132{ }^{\circ} \mathrm{C}$ for $\mathbf{A}$ and $135^{\circ} \mathrm{C}$ for $\mathbf{B}$. Stripes parallel to the rubbing direction in Figure 2(b and f) at $118{ }^{\circ} \mathrm{C}$ for $\mathbf{A}$ and $116^{\circ} \mathrm{C}$ for $\mathbf{B}$ are characteristic of the twist-bend nematic phase. Figure 2 (c) and (g) at $109.3{ }^{\circ} \mathrm{C}$ for $\mathbf{A}$ and $107^{\circ} \mathrm{C}$ for $\mathbf{B}$ show the texture during (A) and $0.5^{\circ} \mathrm{C}$ below (B) phase transition to a smectic phase, respectively. During the transition twisted ropelike dendrites grow with the widths of the ropes and their final direction corresponding to the stripes of the $\mathrm{N}_{\mathrm{TB}}$ phase, as seen in Figure 2(c). After the transition being completed, the texture is characterized by twisted stripes running parallel to the stripes observed in the $\mathrm{N}_{\mathrm{TB}}$ phase. About $4-5^{\circ} \mathrm{C}$ below the transition the stripes fade away, as seen in Figs. $2(\mathrm{~d})$ and $(\mathrm{h})$ at $104^{\circ} \mathrm{C}$ and $102^{\circ} \mathrm{C}$ for A and B, respectively. 

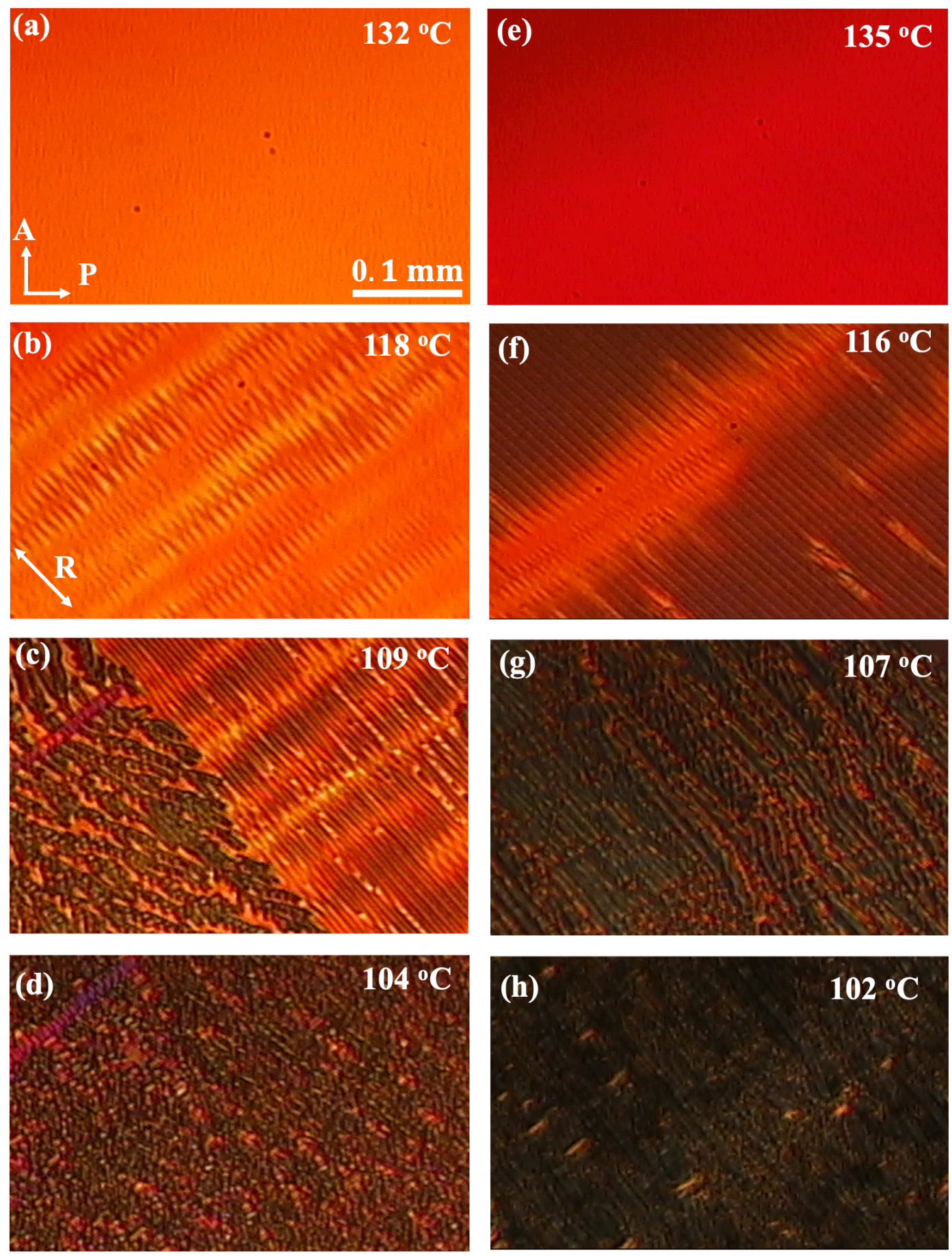

Figure 2: Polarized optical microscopy (POM) textures of $5 \mu$ m, planar aligned $\boldsymbol{A}$ (left) \& $\boldsymbol{B}$ (right) samples. Top row ( $a$ and e): nematic phase. Second row (b and f): $N_{T B}$ phase. Stripes are parallel to the rubbing direction (white double headed arrow in (b)). Third row (c and g): During and $0.5^{\circ} \mathrm{C}$ below $N_{T B}-S m X$ transition, respectively. The textures are characterized by twisted ropes running parallel to the rubbing direction. Bottom row (d and h): Smectic textures about $5^{\circ} \mathrm{C}$ below the $N_{T B}-S m X$ transition where the rope textures fade away. 


\section{C. $\quad$ Small Angle X-ray Scattering (SAXS)}

Small angle X-ray scattering (SAXS) measurements were carried out at beamline 7.3.3 of the Advanced Light Source (ALS) at Lawrence Berkeley National Laboratory with $10 \mathrm{KeV}$ x-ray energy. Both samples of $\mathbf{A}$ and $\mathbf{B}$ were loaded in $2 \mathrm{~mm}$ diameter quartz capillaries and placed in a customized Instec hot stage equipped with SmCo magnets, which produced a 1.5 Tesla magnetic field across the sample to align the nematic director. 2D SAXS patterns were recorded on a Pilatus $2 \mathrm{M}$ detector (Dectris, Inc.) at $2 \mathrm{~m}$ distance from the sample. Each sample was heated to the isotropic phase and cooled slowly into the liquid crystal phases, where SAXS data were taken.
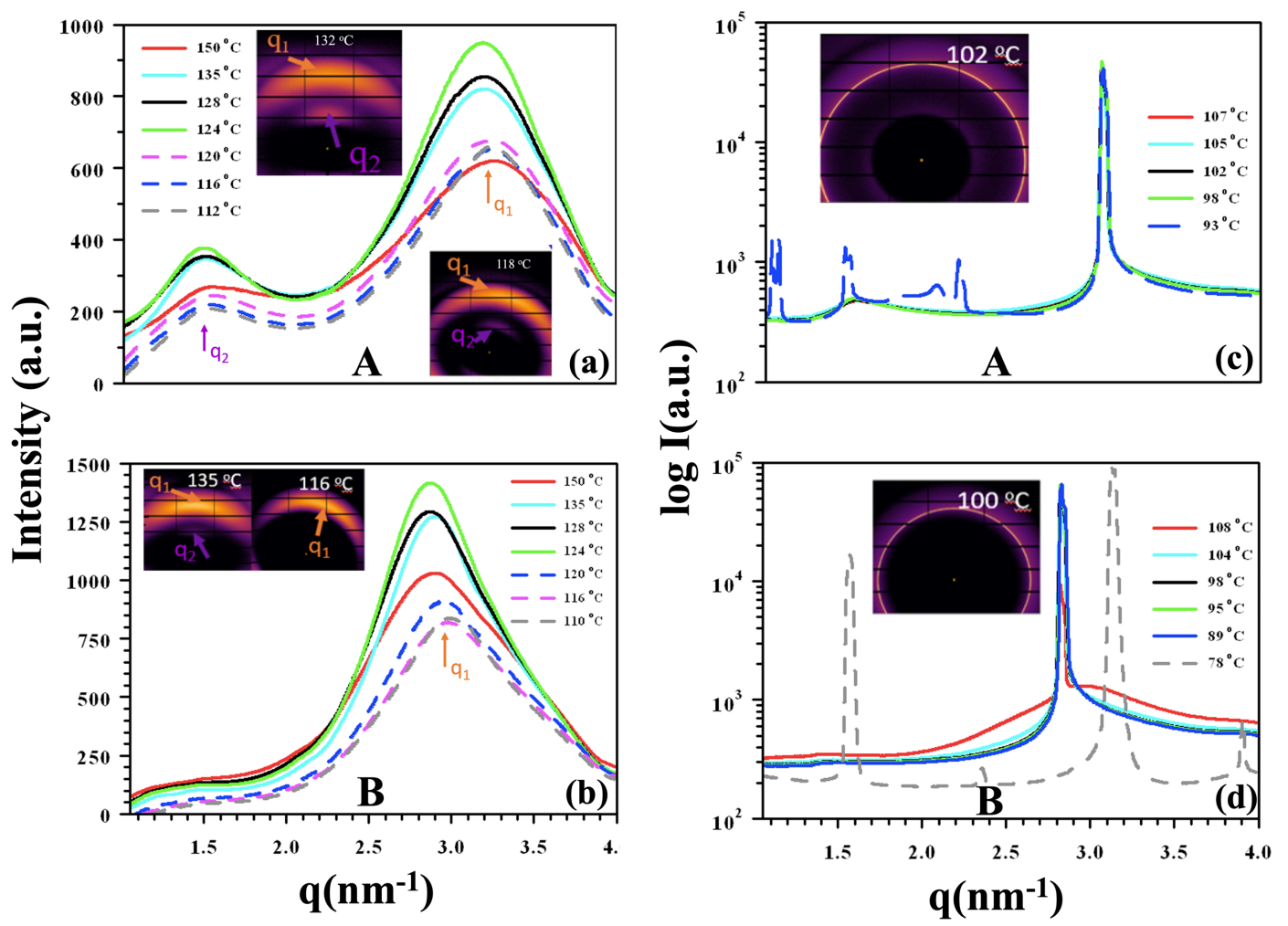

Figure 3: Scattered intensity vs wavenumber (q) in $1<q<4 \mathrm{~nm}^{-1}$ range at selected temperatures of $\boldsymbol{A}$ (top: $a, c)$ and $\boldsymbol{B}$ (bottom: $b, d)$. Left side ( $a$ and $b$ ): $q$-dependence of the scattered intensity in the $N$ (solid lines) and $N_{T B}$ (dashed lines) phases. Insets: $2 D$ images at $132^{\circ} \mathrm{C}$ and $118^{\circ} \mathrm{C}$ of $\boldsymbol{A}$ (a) and at $135^{\circ} \mathrm{C}$ and $116^{\circ} \mathrm{C}$ of $\boldsymbol{B}(b)$. Dark lines are edges of the detector array. Right side (c and d): $q$ dependence of the scattering intensity in the SmA (solid lines) and crystal (dashed lines) phases. Insets: $2 D$ patterns of SmA phase of $\boldsymbol{A}(c)$ and $\boldsymbol{B}(d)$.

As seen in Figure 3(a), A exhibits two diffuse peaks both in the $N$ (solid lines) and $N_{T B}$ (dashed lines) phases. These peaks are centered at $q_{1} \sim 3.25 \mathrm{~nm}^{-1}$ and $q_{2} \sim 1.53 \mathrm{~nm}^{-1}$, corresponding 
to $d_{1} \sim 1.93 \mathrm{~nm}$ and $d_{2} \sim 4.10 \mathrm{~nm}$ spatial periodicities. As the molecular length is estimated to be $4.0 \mathrm{~nm}$ in the fully extended, bent conformation, the presence of two peaks indicates that the separation of the molecules is not only between aromatic and aliphatic groups, but there is also a separation between aliphatic terminal chains and spacers, i.e., both dimer-dimer and monomermonomer interactions occur. The intensity at $q_{1}$ is about 4 times larger than at $q_{2}$, representing more populated monomer-monomer (periodicity $d_{l}$, slightly smaller than half length of the dimer) than dimer-dimer (periodicity $d_{2}$ corresponding to the dimer length) associations. ${ }^{22}$ This ratio is surprisingly different from the parent molecule where only one peak, corresponding to dimerdimer associations, was found in the $N_{T B}$ phase. ${ }^{46}$ From the full width at half maxima $(F W H M)$, we estimate the correlation length of the monomer-monomer association to be $\xi=1 / \mathrm{FWHM} \sim 8-10$ $n m$, corresponding to 2-3 molecular length. For B (Figure 3(b)) with the longer spacer, the peak with $q_{2}$ is basically missing, indicating only monomer-monomer type associations with $q_{1} \sim 2.88$ $\mathrm{nm}^{-1}$ corresponding to $d_{1} \sim 2.1 \mathrm{~nm}$ periodicity. This differs from the parent DTC5C9 system, where peaks corresponding to both monomer and dimer associations were detected. ${ }^{47}$ This is likely due to the longer spacer that affords greater conformational freedom, disfavoring the dimer-dimer correlations. Figures 3(c) and (d) show that on cooling below $108^{\circ} \mathrm{C}$ for $\mathbf{A}$ and $107^{\circ} \mathrm{C}$ for $\mathbf{B}$ the peaks at $q_{1}$ sharpen considerably, and the intensity increases by over three orders of magnitude indicating the formation of a smectic phase with layer periodicity being approximately half of the molecular length. For A only, there is an additional diffuse peak at $q_{2}$ (because this peak is so week, it is only discernible in a semi-logarithmic plot). This additional peak indicates some axially polar dimer-dimer associations within the apolar arrangement of molecules with head-tail symmetry. The peak position of $q_{1}$ in both $\mathbf{A}$ and $\mathbf{B}$ is basically independent of the temperature in the smectic phase.

As Figure 4(a) shows, the temperature dependences of the periodicities corresponding to the short-range monomer-monomer associations in the $N$ and $N_{T B}$ phases and of the layer spacing in the smectic phase are similar in the two materials. In the nematic phase, the periodicity of the molecular associations increases on cooling, reaches a plateau about $10^{\circ} \mathrm{C}$ above the $N-N_{T B}$ transition, and then decreases (especially for $\mathbf{A}$ with shorter spacer) before reaching the transition. Such pretransitional behavior has been observed for a number of dimers ${ }^{48-50}$ and can be understood as a tilt of the molecular axis in fluctuating $N_{T B}$ domains with respect to the nematic director. ${ }^{51}$ The 
decrease of the periodicity continues below the $N-N_{T B}$ transition, but more weakly in $\mathbf{A}$, and then starts to increase about $8^{\circ} \mathrm{C}$ above the transition to the smectic phase. In case of $\mathbf{B}$, the period of short-range monomer-monomer associations decreases from $2.19 \mathrm{~nm}$ to $2.08 \mathrm{~nm}$, suggesting a tilt of $\theta \approx \cos ^{-1}(2.08 / 2.19) \sim 18^{\circ}$. This value is very similar to that found in the non-sulfur containing analogue, DTC5C9, as determined by the ratios of the smallest and largest periodicities of the cybotactic layer spacing measured above and below the $\mathrm{N}-\mathrm{N}_{\mathrm{TB}}$ transition, respectively. ${ }^{22}$ However, this apparent tilt is much smaller than that obtained using the ratio of the measured cybotactic layer spacing and the helical contour length between the centers of two mesogens, which yielded $\theta=29^{\circ}$ for the DTC analogues. ${ }^{37}$

The peak position of $q_{1}$ in both $\mathbf{A}$ and $\mathbf{B}$ is basically independent of the temperature in the smectic phase. This is strikingly different from the results for the parent DTC5C7 system where a strong dependence of the layer spacing was detected and where additionally the formation of an oblique 2D lattice was found. In the smectic phase the layer spacings are $2.06 \mathrm{~nm}$ and $2.17 \mathrm{~nm}$ in $\mathbf{A}$ and $\mathbf{B}$, respectively. This indicates that there is almost no separation between terminal groups and spacers, and the assembly has preferentially shifted to a structure where molecules span two layers. These values correspond to roughly half of the dimer lengths, indicating apolar and random positions of the flexible spacers in neighbor molecules in a layer, as depicted in the inset to Figure 4(a). The difference in the layer spacings for $\mathbf{A}$ and $\mathbf{B}$ is consistent with the different flexible spacer lengths. Interestingly, for $\mathbf{A}$ the layer spacing is significantly higher than the lengthwise monomer-monomer separation in the $\mathrm{N}$ and $\mathrm{N}_{\mathrm{TB}}$ phases, whereas in $\mathbf{B}$ they are much closer. In analogy to the phase assignment used for bent core molecules, the phase can be assigned as an interdigitated SmA phase. This is also in line with the observed temperature invariance of the layer spacing. ${ }^{31}$ 


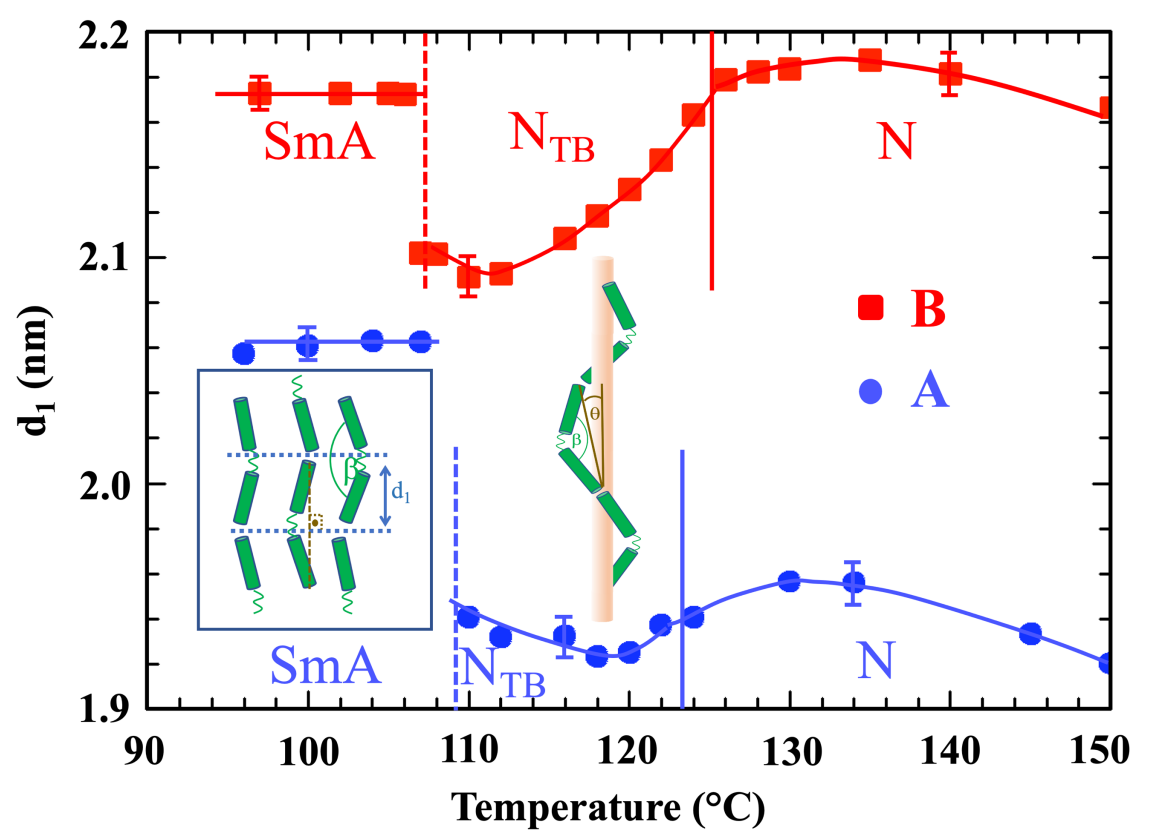

(a)

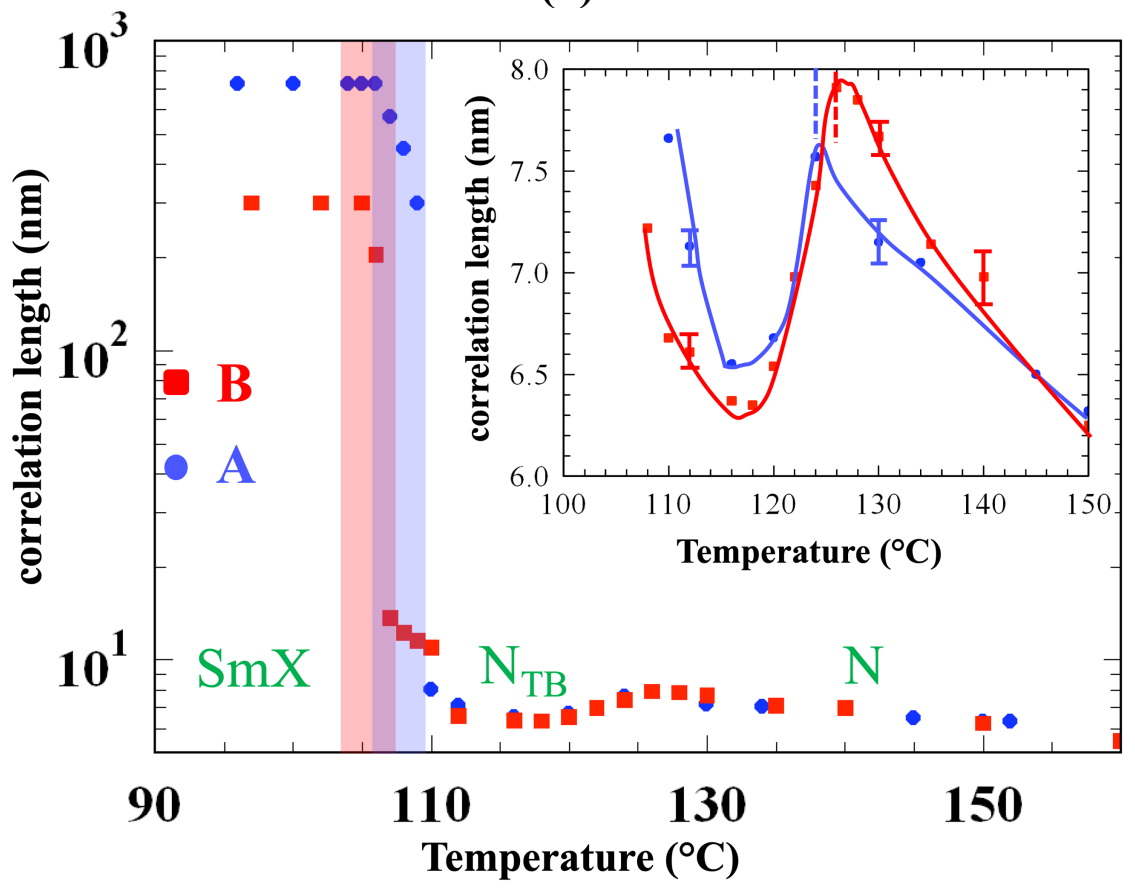

(b)

Figure 4: Temperature dependences of the periodicity $d_{1}$ and the positional correlation length of $\boldsymbol{A}$ (blue dots) and $\boldsymbol{B}$ (red squares). (a): The periodicity calculated from the peak positions of the $q_{1}$ peaks. Box shows the suggested molecular packing in the smectic X phase; (b) Correlation length in logarithmic scale. Inset shows the correlation length in linear scale only in the $N$ and $N_{T B}$ phases. Lines are guides to eye. Light blue and red highlights indicate smectic ranges for $\boldsymbol{A}$ and $\boldsymbol{B}$ where the rope-like texture resembling to the $N_{T B}$ texture was observable in POM studies (see Figure $2 d$ and $g$ ). 
As seen in Figure 4(b), for both materials the correlation length decreases upon the N-N $\mathrm{N}_{\mathrm{TB}}$ transition from $7.6 \mathrm{~nm}$ to $6.6 \mathrm{~nm}$ for $\mathbf{A}$ and $8.0 \mathrm{~nm}$ to $6.3 \mathrm{~nm}$ for $\mathbf{B}$, indicating a decrease in the short-range positional order. This is the same trend observed for the $\mathrm{DTCnCm}$ materials without sulfur atom. ${ }^{22}$ Due to the underlying smectic phase, the correlation length increases gradually in the lower temperature range of the $\mathrm{N}_{\mathrm{TB}}$ phase, then grows sharply over a $\sim 4^{\circ} \mathrm{C}$ range (shown by light blue and red highlights in Fig. 4(b)) to over $700 \mathrm{~nm}$ and $300 \mathrm{~nm}$ in the smectic phase of $\mathbf{A}$ and $\mathbf{B}$, respectively.

Our SAXS results provide useful information on the orientational and positional order. However, they do not give any information about the heliconical pitch in the $\mathrm{N}_{\mathrm{TB}}$ phase; the latter may be determined from Tender Resonant X-ray Scattering.

\section{D. $\quad$ Tender Resonant X-ray Scattering (TReXS)}

TReXS measurements were carried out at beamline 5.3.1 in the Advanced Light Source (ALS), Lawrence Berkeley National Laboratory. A and $\mathbf{B}$ were melt-loaded in between two silicon nitride membranes. Birefringence color indicated sample thicknesses in the range of 5-10 $\mu \mathrm{m}$. The samples were attached to a home-made heater which were sealed inside a helium chamber on the beamline. All samples were initially annealed in the isotropic state to remove heat history and defects. The X-ray beam energy was set at the sulfur K-edge by a channel cut double-bounce silicon monochromator. ${ }^{39}$ A $2 \mathrm{D}$ detector (Pilatus 300K, Dectris, Inc) was used to collect the scattering patterns at a sample-detector distance of $393 \mathrm{~mm}$. The beam center and the sample-todetector distance were calibrated using silver behenate and the smectic A phase of 8CB. All TReXS data presented are measured on cooling at $1^{\circ} \mathrm{C}$ rate after the samples heated to the isotropic phase. No well-defined features (rings/peaks) are seen in the scattering from the nematic phase. Intensity vs scattering wavenumber $(q)$ curves for $\mathbf{A}$ and $\mathbf{B}$ were obtained from the $2 \mathrm{D}$ scattering patterns shown in the inset of Figure 5 using the Nika software package. ${ }^{52}$

The heliconical pitch $(p)$ was calculated from the peak positions as $p=2 \pi / q$. The temperature dependence of the pitch for both materials are plotted in Figure 5. After initially decreasing rapidly below the $\mathrm{N}-\mathrm{N}_{\mathrm{TB}}$ phase transition, $p$ approaches an asymptotic value far from the transition, as observed for other dimers by RSoXS ${ }^{34-37}$ and TReXS ${ }^{39-43}$ techniques. Interestingly the temperature ranges where the nanoscale pitch was detected are $16.5^{\circ} \mathrm{C}$ and $22^{\circ} \mathrm{C}$ for $\mathbf{A}$ and $\mathbf{B}$, respectively. These values are $3.3^{\circ} \mathrm{C}$ and $4.4^{\circ} \mathrm{C}$ larger than what we found by POM 
and SAXS measurements. As the $\mathrm{N}-\mathrm{N}_{\mathrm{TB}}$ transition temperatures measured by the different techniques are the same within a degree, the difference is due to the fact that the nanoscale pitch disappears about $3-4^{\circ} \mathrm{C}$ below the transition to the smectic phase observed by SAXS and POM. This structure can also explain the twisted rope-type texture that appears in the POM images right below the $\mathrm{N}_{\mathrm{TB}}-\mathrm{SmX}$ transition with width and direction matching the stripe texture of the $\mathrm{N}_{\mathrm{TB}}$ phase (see Figure 2(c) and (g)). These observations strongly indicate that the nanoscale pitch survives in the top range of the smectic phase.

The smectic layer periodicity of $\sim 2 \mathrm{~nm}$ is not detected in our TReXS measurements, because the corresponding wavenumber falls outside the range of our detector. After further cooling to the crystal phase, new peaks appear, corresponding to spatial periodicities of approximately 4,6 and $8 \mathrm{~nm}$, indicating that they are harmonics of the $2 \mathrm{~nm}$ periodicity of the monomers.

The temperature dependence of pitch data measured in the $\mathrm{N}_{\mathrm{TB}}$ phase can be fitted to the expression

$$
p(T)=p_{o}+\Delta p \cdot\left(1-T / T_{C}\right)^{-\gamma}
$$

Here $p_{\mathrm{o}}$ is the asymptotic pitch value very far from the critical temperature $T_{C}$, which is slightly $\left(\sim 1^{\circ} \mathrm{C}\right)$ larger than the $N-N_{T B}$ phase transition temperature $T_{T B}$. The parameter $\Delta p$ is the coefficient of the temperature dependent term, and $\gamma$ is the critical exponent of the temperature dependent term. Since we do not have data up to $\mathrm{T}_{\mathrm{C}}$, the four parameters in Eq. 1 appear not sufficiently independent to reliably determine them by least squares fitting; indeed, it is possible to obtain reasonable looking fits with $\gamma$ ranging from 0.2 to 1 . For this reason, motivated by predictions of macroscopic mean-field theories ${ }^{23,30,31}$, we elected to fix the value of $\gamma$ to 0.5 . Figure 5 shows fits to Eq. (1) for this fixed value. The fits give $p_{\mathrm{o}}=6.76 \mathrm{~nm}$ and $6.35 \mathrm{~nm}, \Delta p=0.50 \mathrm{~nm}$ and $1.07 \mathrm{~nm}$ and $T_{C}=124.4^{\circ} \mathrm{C}$ and $127.4^{\circ} \mathrm{C}$ for $\mathbf{A}$ and $\mathbf{B}$, respectively. The fitted critical temperature values are about $2^{\circ} \mathrm{C}$ higher than what we observed by POM measurements based on the appearance of the micrometer-range stripes due to the Helfrich-Hurault-type instability. ${ }^{48}$ This difference is reasonable, since these stripes usually appear $1-2^{\circ} \mathrm{C}$ below the actual transition, where the decrease of the heliconical pitch is sufficiently large. Similarly, the obtained $\Delta p$ values are also reasonable, as the ratios of the values of $\Delta p$ for $\mathbf{B}$ and $\mathbf{A}$ are approximately the same as the ratio of the 
corresponding changes in the spatial correlation lengths (see inset of Figure 4(b)) and of the variation of the pitch in the measured range. These indicate that the choice of the exponent $\gamma=0.5$ predicted by mean-field theory is a reasonably good. However, the fact that $p_{\mathrm{o}}$ is smaller for the longer molecule B than for A suggests that the exponent $\gamma=0.5$, expected only to describe the pretransitional temperature dependence of $p$, does not hold far from the transition.

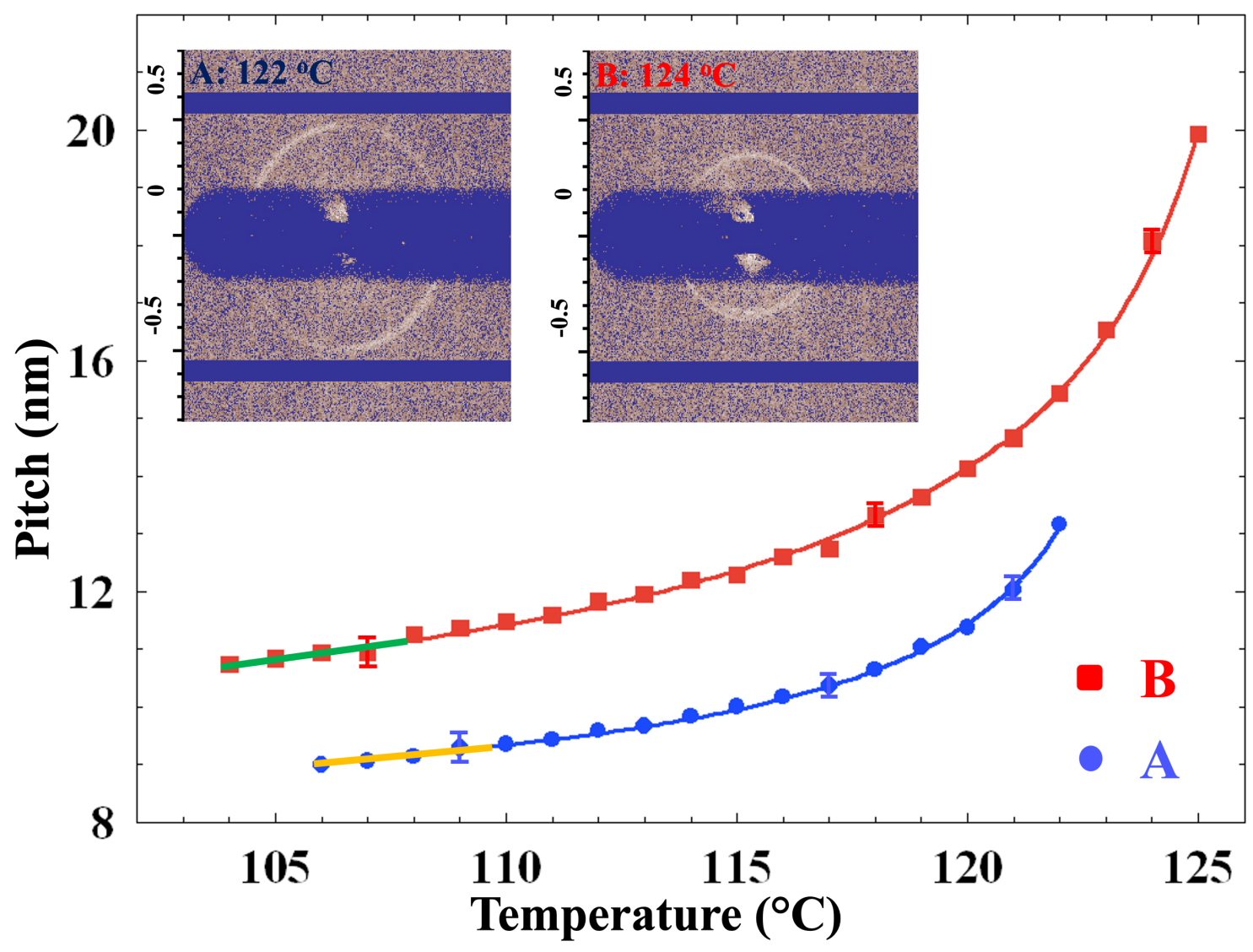

Figure 5: Temperature dependences of the heliconical pitch of $\boldsymbol{A}$ (blue dots) and $\boldsymbol{B}$ (red squares). Solid lines with matching colors correspond to fit equation given in Eq. (1) with $\gamma=0.5$ and best fit parameters $p_{o}=6.76 \mathrm{~nm}$ and $6.35 \mathrm{~nm}$ and $\Delta p=0.88 \mathrm{~nm}$ and $1.88 \mathrm{~nm}$ for $\boldsymbol{A}$ and $\boldsymbol{B}$, respectively. Pictures show the 2-dimensional scattering profiles for $\boldsymbol{A}$ and $\boldsymbol{B}$ at $122^{\circ} \mathrm{C}$ and $124^{\circ} \mathrm{C}$, respectively. Scale numbers of the pictures are in $\mathrm{nm}^{-1}$ unit. Orange and green lines indicate the range where the helix coexist with the smectic order for $\boldsymbol{A}$ and $\boldsymbol{B}$, respectively.

The temperature dependence of the correlation length of the heliconical order for $\mathbf{A}$ and $\mathbf{B}$ is shown in Figure 6 (a). The correlation length was calculated from the full width at half maxima (FWHM) of the peaks as $\xi=1 /$ FWHM (see inset of Figure 6(a)). The FWHM was determined by fitting the data for the scattered intensity vs $q$ to a Gaussian, $I(q)=a+b \cdot \exp \left(-\left(q-q_{o}\right)^{2} / \Delta q^{2}\right)$, and 
then taking FWHM= $2 \Delta q(\ln 2)^{1 / 2}$. The values of $\xi$ observed near the transition at $T=T_{T B}$ are only slightly larger than of the measured pitch at $T_{T B}$, corresponding to the situation where the heliconical structure just barely can form. For both materials $\xi$ increases sharply below $T_{T B}$ to 55 $\mathrm{nm}$ and $44 \mathrm{~nm}$ for $\mathbf{A}$ and $\mathbf{B}$, respectively, which are 4-5 times longer than the heliconical pitch. The increase stops for $\mathbf{A}$ at around $116^{\circ} \mathrm{C}$, then the correlation length decreases continuously to about $45 \mathrm{~nm}$. For $\mathbf{B}$ there is a weak local minimum at $114^{\circ} \mathrm{C}$, then the correlation length increases to about $48 \mathrm{~nm}$. For both materials the peak width decreases (i.e., the apparent correlation length increases) as the peak height drops before the heliconical structure disappears at $106^{\circ} \mathrm{C}$ for $\mathbf{A}$ and $103^{\circ} \mathrm{C}$ for $\mathbf{B}$.

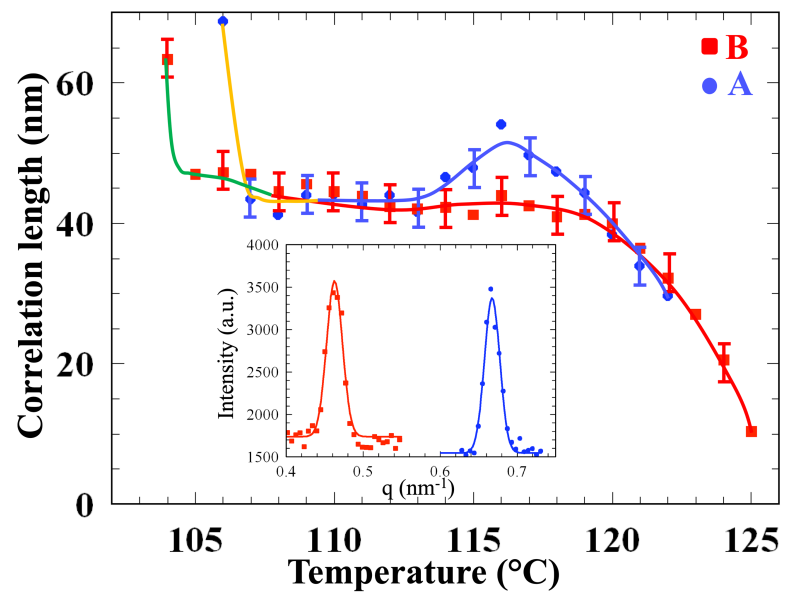

(a)

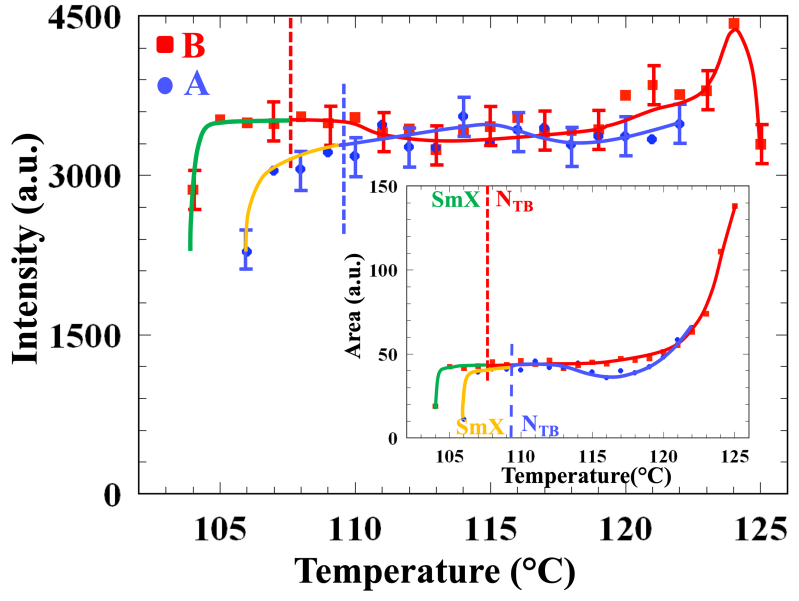

(b)

Figure 6: Temperature dependences of the correlation length of the heliconical bond order (a) and the peak intensities (b) for $\boldsymbol{A}$ and $\boldsymbol{B}$. Inset of (a) shows representative intensity vs $q$ graphs with Gaussian fit to the measured data. Lines are guidance for the eye. Inset of (b) shows the areas below the peaks. Orange and green lines indicate the range where the helix coexist with the smectic order for $\boldsymbol{A}$ and $\boldsymbol{B}$, respectively.

The temperature dependences of the maximum intensities of the peaks and the area below the peaks are shown in the main pane and in the inset of Figure 6(b). For both materials the intensity is almost constant over the entire range except for a small maximum at $115^{\circ} \mathrm{C}$ for $\mathbf{A}$ and at $124^{\circ} \mathrm{C}$ for B. Importantly the intensity does not decrease in the last $3-4^{\circ} \mathrm{C}$ where the smectic phase has already formed, but only within $1^{\circ} \mathrm{C}$ before the peak disappears. Note, these correlation lengths are an order of magnitude smaller than those estimated for the prototypical cyano-biphenyl type dimer $C B 7 C B$ from FFTEM measurements. ${ }^{6,26}$ 


\section{Discussion}

One of the key observations of our experiments is that on cooling the achiral dimers through the $N-N_{T B}$ phase transition the correlation length of the spatial periodicity decreases, while the heliconical orientational order becomes more correlated. This shows that neither the molecular chirality nor spatial correlation is needed for the formation of the helical structure, and that the heliconical order even suppresses the spatial correlations. Formation of the $N_{T B}$ phase with reduced short-range positional and quasi long-range heliconical orientation order is clearly the result of the molecular bend favored by the odd-membered flexible linkage. Such molecules can fill the space most effectively when twist and bend deformations couple ${ }^{33}$, leading to the substantial heliconical correlations lengths we have observed. The formation of the heliconical order locks the molecular twist-bend conformation, i.e., it makes the linkage effectively rigid. This suppresses the entropic penalty of packing the flexible linkage next to a rigid arm, therefore favoring positional disorder as was found in this work. Such a mechanism for the tight pitch of the twist-bend nematic liquid crystal phase is therefore very different from that of molecularly chiral systems such as proteins and DNA that require both molecular chirality and more significant positional order.

The observation that the heliconical structure survives in the upper $3-4^{\circ} \mathrm{C}$ range of the smectic phase forming below the $N_{T B}$ phase, shows the strength of the well-developed heliconical order is comparable with the long-range positional order. Here we note that the coexistence of smectic layering and a heliconical structure has been already been reported by Abberly et al ${ }^{53}$ in dimeric mixtures that exhibited $\mathrm{N}$ and $\mathrm{SmA}$ phases but no $\mathrm{N}_{\text {тв }}$ phase and was designated as $S m C_{T B}$ phase. In our case, the twist-bend type smectic phase is observed below the $\mathrm{N}_{\text {TB }}$ phase and the heliconical structure disappears about $4{ }^{\circ} \mathrm{C}$ below the transition. SAXS measurements reveal that the intensity of the scattering peak and the spatial correlation length increase abruptly in this range and reach maxima before the heliconical structure disappears (see Figure 4(b)) without causing any observable change in the layer periodicity. At the same time, the TReXS peak intensities are almost constant and decrease abruptly only when the heliconical structure disappears. These indicate that both the smectic layers and the heliconical structure fill the entire volume simultaneously without affecting each other. This suggests the formation of a $S m A_{T B}$-type structure where the rigid units of the dimers are tilted with respect to the layer normal to allow for the 
bending of the dimers, and the tilt direction rotates following the heliconical order. Such a structure implies zero cone angle $\theta$ of dimers with respect to the helical axis. Indeed, the temperature dependences of both the positional periodicity and correlation length (see Figure 4) indicate the cone angle reaches a maximum at around $116^{\circ} \mathrm{C}-118^{\circ} \mathrm{C}$ and decreases below this range. In the same temperature range, the correlation length of the heliconical order has a maximum (more pronounced for $\mathbf{A}$ ), indicating that the correlation length of the heliconical order is influenced by the tilt of the molecular planes with respect to the heliconical axis. On the other hand, the pitch continuously decreases until it disappears. Even more striking is that the pitch value has no jump at the transition to the $\operatorname{SmA} A_{T B}$ structure where the cone angle drops to zero.

These observations mean that the value of the pitch is not influenced much by the heliconical cone angle. This is in variance with the "double-helical tiled chain structure" model proposed by Tuchband et $\mathrm{al}^{35}$ based on experimental results on $\mathrm{CB} 7 \mathrm{CB}$, the compound for which the $\mathrm{N}_{\text {TB }}$ phase was first demonstrated. In that material, assuming zero overlap between neighbor molecules, the pitch was found to be related to the cone angle $\theta$ as

$$
p(T)=2 \pi R_{m o l} \cos \theta=k_{0} \cdot L \cdot \cos \theta
$$

where $R_{m o l}$ is the curvature radius of the bent molecules, $L$ is the contour length of the dimer, and $k_{0}$ is the number of molecules making one full turn of the helix. Applying this equation to our materials, we find that even assuming as large cone angle $\left(\theta=29^{\circ}\right)$ as was measured for the DTC analogs $s^{37}$, the variation of the pitch for $\mathbf{B}$ between $125^{\circ} \mathrm{C}$ and $118^{\circ} \mathrm{C}$ should be less than $2.6 \mathrm{~nm}$, in contrast to the measured $9 \mathrm{~nm}$ (see Figure 5). A similar discrepancy was noticed by Cruickshank et al ${ }^{42}$ for several cyano-terminated sulfur containing materials. They proposed that the parameter $k_{0}$ increases as the temperature approaching the $\mathrm{N}_{\mathrm{TB}}-\mathrm{N}$ transition. Implicitly this means that the molecular bend angle $\beta$ decreases on cooling below the $\mathrm{N}_{\text {тв}}-\mathrm{N}$ transition. Similarly, the observation that $p\left(T_{T B}\right)$ is about twice as large for $\mathbf{B}$ with $n=11$ carbons in the flexible linker than for $\mathbf{A}$ with $n=7$, cannot be understood simply by the difference between contour lengths $L_{\boldsymbol{B}}$ and $L_{A}$. Since $L_{B}-L_{A}$ is equal to the length of 4 carbon-carbon bonds $\left(4 \cdot 0.154 \mathrm{~nm} \cdot \cos 60^{\circ} \approx\right.$ $0.31 \mathrm{~nm})$, it gives less than a $10 \%$ increase. The observed $p_{B}\left(T_{T B}\right) / p_{A}\left(T_{T B}\right) \sim 1.7$ can be explained only if we assume $\beta_{A}\left(T_{T B}\right) / \beta_{B}\left(T_{T B}\right) \sim 1.5$. This is reasonable since $\beta$ is expected to decrease with 
increasing length of the spacer due to the flexibility and large number of configurations of the linkage.

As we argued above, the helical structure is due to the coupling between the molecular bend and twist, so the pitch, which is inversely proportional to the twisting power, should decrease as the bend angle decreases on cooling. Such a decrease overcomes the effect of the tilt, explaining the continuous decrease even when the tilt reaches maximum. In fact, the role of the tilt appears to be negligible when the smectic layers form and the cone angle drops to zero. Since the layer spacing was found to be practically constant, the molecular bend angle should be also basically independent of temperature when the layers form. In spite of this, the surviving heliconical pitch slightly decreases, indicating an increasing coupling between the bend and twist on cooling.

Finally, the decrease of the spatial correlation length of the monomer-monomer aggregates below the transition to the $\mathrm{N}_{\text {TB }}$ phase, and the onset of the smectic layering in the lower part of this phase, indicate a variation of the molecular overlap between two molecules along the helical axis. Defining an overlap parameter as $J=l / L$, where $l$ is the length of molecular overlap and $L$ is the contour length of the dimers, for completely zero positional order $J$ randomly varies between 0 and 1 with $\langle J\rangle=1 / 2$, while in the $\operatorname{SmA}$ phase with periodicity about half of the dimer length $J$ is uniform and $J<1 / 2$.

\section{Conclusion}

To summarize, we have measured the nanostructure of two novel sulfur containing dimer materials both by hard Small Angle X Ray Scattering that is sensitive to positional order and by resonant tender X-ray scattering that can detect the heliconical bond order. Our most significant observations are the following: (a) On cooling the dimers through the $N-N_{T B}$ phase transition the correlation length of the spatial periodicity drops, while the heliconical orientational order becomes more correlated. (b) The heliconical pitch is observable even in the upper $3-4^{\circ} \mathrm{C}$ range of the underlying smectic phase. (c) The temperature dependences of the heliconical pitch show stronger variation near the $\mathrm{N}-\mathrm{N}_{\text {ТВ }}$ transition than in prototypical CBnCB-type dimers.

We proposed that the coupling between the temperature dependent molecular bend and twist may account for the observed temperature variation and the spacer length dependence of the heliconical pitch. 
The observed coexistence of smectic layering and heliconical order - both having periodicities on the scale of the molecular length - is consistent with a SmA $\mathrm{AB}_{\text {тв}}$-type phase, where the rigid units of the dimers are tilted with respect to the layer normal to allow for the bent conformation of the dimers, but the tilt direction rotates along the heliconical axis. However, further and more comprehensive experiments are needed to confirm a distinct smectic phase with the twist-bend orientational nanostructure in the dimers investigated here.

Our results demonstrate the value of employing multiple structural probes in order to illuminate the complex interplay between molecular shape, molecular flexibility, and intermolecular packing that governs the microscopic structure of liquid crystalline states, such as the twist-bend phase, that feature novel, nanoscale modulations of the molecular arrangements. The combination of quasi long-range helical orientational order and the strongly varying positional order in these systems represents a particular challenge to structural determination.

\section{Acknowledgement}

This research was supported by the National Science Foundation under grant DMR-1904167 and by (UK) EPSRC project EP/M015726/1. We acknowledge use of Beamlines 7.3.3 and 5.3.1 of the Advanced Light Source supported by the Director of the Office of Science, Office of Basic Energy Sciences, of the U.S. Department of Energy under contract no. DE-AC02-05CH11231.

C. Feng and R. Saha equally contributed to the paper.

\section{References}

1 L. C. PAULING, R. B. Corey and H. R. BRANSON, PNAS, 1951, 37, 205-211.

2 P.-G. de Gennes, The Physics of Liquid Crystals, Clarendon Press, Oxford, 2nd Editio., 1974, vol. Chapter 7.

3 J. W. Emsley, G. R. Luckhurst, G. N. Shilstone and I. Sage, Mol. Cryst. Liq. Cryst., 1984, 102, 223-233.

$4 \quad$ P. J. Barnes, A. G. Douglass, S. K. Heeks and G. R. Luckhurst, Liq. Cryst., 1993, 13, 603613.

5 M. Cestari, E. Frezza, A. Ferrarini and G. R. Luckhurst, J. Mater. Chem., 2011, 21, 12303.

6 V. Borshch, Y.-K. Kim, J. Xiang, M. Gao, A. Jakli, V. P. Panov, J. K. Vij, C. T. Imrie, M.

G. Tamba, G. H. Mehl and O. D. Lavrentovich, Nat. Commun., 2013, 4, 2635-1-8. 
7 K. Adlem, M. Čopič, G. R. Luckhurst, A. Mertelj, O. Parri, R. M. Richardson, B. D. Snow, B. A. Timimi, R. P. Tuffin and D. Wilkes, Phys. Rev. E, 2013, 88, 022503.

8 J. W. Emsley, M. Lelli, G. R. Luckhurst and H. Zimmermann, Phys. Rev. E, 2017, 96, 062702 .

9 M. Cestari, S. Diez-Berart, D. A. Dunmur, A. Ferrarini, M. R. De La Fuente, D. J. B. Jackson, D. O. Lopez, G. R. Luckhurst, M. A. Perez-Jubindo, R. M. Richardson, J. Salud, B. A. Timimi and H. Zimmermann, Phys. Rev. E, 2011, 84, 031704.

10 B. Robles-Hernández, N. Sebastián, M. R. De La Fuente, D. O. López, S. Diez-Berart, J. Salud, M. B. Ros, D. A. Dunmur, G. R. Luckhurst and B. A. Timimi, Phys. Rev. E - Stat. Nonlinear, Soft Matter Phys., 2015, 92, 062505.

11 Y. Arakawa, K. Komatsu and H. Tsuji, New J. Chem., 2019, 43, 6786-6793.

12 Z. Parsouzi, G. Babakhanova, M. Rajabi, R. Saha, P. Gyawali, T. Turiv, H. Wang, A. R. Baldwin, C. Welch, G. H. Mehl, J. T. Gleeson, A. Jakli, D. Lavrentovich and S. Sprunt, Phys. Chem. Chem. Phys., 2019, 15-19.

13 C. T. Archbold, E. J. Davis, R. J. Mandle, S. J. Cowling and J. W. Goodby, Soft Matter, $2015,11,7547$.

14 A. A. Dawood, M. C. Grossel, G. R. Luckhurst, R. M. Richardson, B. A. Timimi, N. J. Wells and Y. Z. Yousif, Liq. Cryst., 2016, 43, 2-12.

15 C. T. Archbold, R. J. Mandle, J. L. Andrews, S. J. Cowling and J. W. Goodby, Liq. Cryst., 2017, 44, 2079-2088.

16 R. J. Mandle, M. P. Stevens and J. W. Goodby, Liq. Cryst., 2017, 44, 2046-2059.

17 F. F. P. Simpson, R. J. Mandle, J. N. Moore and J. W. Goodby, J. Mater. Chem. C, 2017, 5, 5102-5110.

18 Y. Arakawa, K. Komatsu, S. Inui and H. Tsuji, J. Mol. Struct., 2020, 1199, 126913.

19 K. L. Atkinson, S. M. Morris, F. Castles, M. M. Qasim, D. J. Gardiner and H. J. Coles, Phys. Rev. E, 2012, 85, 12701.

20 M. R. Tuchband, D. A. Patersonb, M. Salamo!nczyk, V. A. Norman, A. N. Scarbrough, E. Forsyth, E. Garciag, C. Wangd, J. M. D. Storey, D. M. Walba, S. Sprunt, A. Jákli, C. Zhu, C. T. Imrie and N. A. Clark, PNAS, 2019, 116, 10698-10704.

21 R. J. Mandle and J. W. Goodby, RSC Adv., 2016, 6, 34885-34893.

22 R. Saha, G. Babakhanova, Z. Parsouzi, M. Rajabi, P. Gyawali, C. Welch, G. H. Mehl, J. 
Gleeson, O. D. Lavrentovich, S. Sprunt and A. Jakli, Mater. Horizons, 2019, 6, 1905-1912.

23 I. Dozov, Eur. Lett., 2001, 56, 247-253.

24 V. Panov, M. Nagaraj, J. Vij, Y. Panarin, A. Kohlmeier, M. Tamba, R. Lewis and G. Mehl, Phys. Rev. Lett., 2010, 105, 167801.

25 C. Meyer, G. R. Luckhurst and I. Dozov, Phys. Rev. Lett., 2013, 111, 067801.

26 D. Chen, J. H. Porada, J. B. Hooper, A. Klittnick, Y. Shen, M. R. Tuchband, E. Korblova, D. Bedrov, D. M. Walba, M. A. Glaser, J. E. Maclennan and N. A. Clark, Proc. Natl. Acad. Sci. U. S. A., 2013, 110, 15931-15936.

27 K. Merkel, A. Kocot, C. Welch and G. H. Mehl, Phys. Chem. Chem. Phys., 2019, 21, 22839-22848.

28 E. T. Samulski, A. G. Vanakaras and D. J. Photinos, ArXiv, 2020, 2006.00069.

29 I. Dozov and G. R. Luckhurst, Liq. Cryst., 2020, 1-18.

30 S. M. Shamid, S. Dhakal and J. V. Selinger, Phys. Rev. E, 2013, 87, 052503.

31 A. Jákli, O. D. Lavrentovich and J. V. Selinger, Rev. Mod. Phys., 2018, 90, 045004.

32 C. Greco, G. R. Luckhurst and A. Ferrarini, Soft Matter, 2014, 10, 9318-9323.

33 A. G. Vanakaras and D. J. Photinos, Soft Matter, 2016, 12, 2208-2220.

34 C. Zhu, M. R. Tuchband, A. Young, M. Shuai, A. Scarbrough, D. M. Walba, J. E. Maclennan, C. Wang, A. Hexemer and N. A. Clark, Phys. Rev. Lett., 2016, 116, 147803.

35 M. R. Tuchband, M. Shuai, K. A. Graber, D. Chen, C. Zhu, L. Radzihovsky, A. Klittnick, L. Foley, A. Scarbrough, J. H. Porada, M. Moran, J. Yelk, E. Korblova, D. M. Walba, A. Hexemer, J. E. Maclennan, A. Matthew and N. A. Clark, arXiv:1703.10787v1, 2017.

36 M. Salamończyk, N. Vaupotič, D. Pociecha, C. Wang, C. Zhu and E. Gorecka, Soft Matter, 2017, 13, 6694-6699.

37 W. D. Stevenson, Z. Ahmed, X. B. Zeng, C. Welch, G. Ungar and G. H. Mehl, Phys. Chem. Chem. Phys., 2017, 19, 13449-13454.

38 B. L. Henke, E. M. Gullikson and J. C. Davis, At. Data Nucl. Data Tables, 1993, 54, 181342.

39 Y. Cao, J. Feng, A. Nallapaneni, Y. Arakawa, K. Zhao, F. Liu and C. Zhu, arXiv, 2019, 1907.11330v2.

40 M. Salamończyk, R. J. Mandle, A. Makal, A. Liebman-Peláez, J. Feng, J. W. Goodby and C. Zhu, Soft Matter, 2018, 14, 9760-9763. 
41 Y. Cao, J. Feng, A. Nallapaneni, Y. Arakawa, K. Zhao, G. H. Mehl, F. Liu and C. Zhu, ArXiv, 2019, 1907.11330, 1-30.

42 E. Cruickshank, M. Salamończyk, D. Pociecha, G. J. Strachan, J. M. D. Storey, C. Wang, J. Feng, C. Zhu, E. Gorecka and C. T. Imrie, Liq. Cryst., 2019, 46, 1595-1609.

43 C. Feng, J. Feng, R. Saha, Y. Arakawa, J. Gleeson, S. Sprunt, C. Zhu and A. Jákli, Phys Rev Res. Rapid Commun., 2020, 2, 032004(R).

44 Y. Cao, C. Feng, A. Jakli, C. Zhu and F. Liu, Giant, 2020, 2, 100018.

45 M. G. Tamba, S. M. Salili, C. Zhang, A. Jakli, G. H. Mehl, R. Stannarius and A. Eremin, RSC Adv., 2015, 5, 11207-11211.

46 N. Sebastián, M.-G. G. Tamba, R. Stannarius, M. R. De La Fuente, M. Salamonczyk, G. Cukrov, J. T. Gleeson, S. Sprunt, A. Jakli, C. Welch, Z. Ahmed, G. H. Mehl and A. Eremin, Phys. Chem. Chem. Phys., 2016, 18, 19299-19308.

47 W. D. Stevenson, H. X. Zou, X. B. Zeng, C. Welch, G. Ungar and G. H. Mehl, Phys. Chem. Chem. Phys., 2018, 20, 25268-25274.

48 P. K. Challa, V. Borshch, O. Parri, S. N. Sprunt, C. T. Imrie, S. N. Sprunt, J. T. Gleeson, O. D. Lavrentovich and A. Jákli, Phys. Rev E, 2014, 89, 060501 (R)-1-5.

49 C. Greco, A. Ferrarini, G. R. Luckhurst, B. A. Timimi and H. Zimmermann, Liq. Cryst., 2018, 45, 2361-2375.

50 J. W. Emsley, M. Lelli, H. Joy, M.-G. G. Tamba and G. H. Mehl, Phys. Chem. Chem. Phys., 2016, 18, 9419-9430.

51 L. M. Heist, E. T. Samulski, C. Welch, Z. Ahmed, G. H. Mehl, A. G. Vanakaras and D. J. Photinos, Liq. Cryst., 2020, 00, 1-16.

52 J. Ilavsky, Appl. Crystallogr., 2012, 45, 324-328.

53 J. P. Abberley, R. Killah, R. Walker, J. M. D. Storey, C. T. Imrie, M. Salamonzyk, C. Zhu, E. Gorecka and D. Pociecha, Nat. Commun., 2018, 9, 228-1-7. 